\title{
Total saponins from Aralia taibaiensis protect against myocardial ischemia/reperfusion injury through AMPK pathway
}

\author{
JIAJIA YAN*, JIALIN DUAN*, XIAOXIAO WU*, CHAO GUO, YING YIN, \\ YANRONG ZHU, TIANXIN HU, GUO WEI, AIDONG WEN and MIAOMIAO XI \\ Department of Pharmacy, Xijing Hospital, Fourth Military Medical University, Xi'an, Shaanxi 710032, P.R. China
}

Received March 13, 2015; Accepted September 24, 2015

DOI: $10.3892 /$ ijmm.2015.2391

\begin{abstract}
It was previously shown that total saponins extracted from Aralia taibaiensis (sAT) have potent antioxidant activities for treating diabetes mellitus and attenuate D-galactose-induced aging. Since diabetes mellitus and aging are closely associated with cardiac dysfunction, particularly ischemic heart disease, sAT may have potential protective activity against myocardial ischemia/reperfusion injury (MI/RI). However, the anti-MI/RI effects of sAT have yet to be examined, and the possible molecular mechanisms remain to be determined. The present study was undertaken to investigate the anti-MI/RI activities of sAT and to elucidate the mechanisms underlying these effects in rats using TUNEL and Hoechst 33258 staining. The results confirmed the cardioprotective effects in vivo and elucidated the potential molecular mechanisms of sAT in vitro. Pretreatment with sAT significantly reduced infarct size, decreased the levels of lactate dehydrogenase and creatine kinase in the serum and blocked apoptosis. In addition, sAT inhibited A/R-induced apoptosis by decreasing DNA strand breaks, caspase-3 activity and cytochrome $c$ release in H9c2 cells. Furthermore, sAT markedly increased the phosphorylation of AMP-activated protein kinase (AMPK) and acetyl CoA carboxylase and elevated the Bcl2/ Bcl-2-associated X protein ratio. These effects were blocked
\end{abstract}

Correspondence to: Professor Miaomiao $\mathrm{Xi}$, Department of Pharmacy, Xijing Hospital, Fourth Military Medical University, 127 Changle West Road, Xi'an, Shaanxi 710032, P.R. China

E-mail: miaomiaoxi2014@163.com

Abbreviations: AAR, ischemic-reperfused area-at-risk; ACC, acetyl CoA carboxylase; AMPK, AMP-activated protein kinase; Bax, Bcl-2-associated $\mathrm{X}$ protein; CK-MB, creatine kinase isoenzyme-MB; DMEM, Dulbecco's modified Eagle's medium; FBS, fetal bovine serum; INF, myocardial infarct size; LDH, lactate dehydrogenase; MI/RI, myocardial ischemia/reperfusion injury; MTT, 3-(4,5-dimethylthiazol-2-yl)-2,5-diphenyl-tetrazolium bromide; sAT, total saponins extracted from Aralia taibaiensis; TTC, 2,3,5-triphenyltetrazolium chloride; TUNEL, terminal deoxynucleotidyl transferase-mediated dUTP nick end-labeling

Key words: total saponins, Aralia taibaiensis, cardioprotection, myocardial ischemia/reperfusion injury, apoptosis, AMP-activated protein kinase pathway by compound $\mathrm{C}$. The results suggested that sAT pretreatment exerts protective effects on myocardial cells in vitro and in vivo against MI/RI-induced apoptosis by activating AMPK pathway.

\section{Introduction}

Ischemic heart disease, particularly acute myocardial infarction, is a leading cause of morbidity and mortality worldwide (1). Immediate restoration of the interrupted blood supply to the heart by thrombolysis or percutaneous transluminal coronary angioplasty is the main mode of treatment (2-4). However, abrupt reperfusion of ischemic myocardium can exacerbate the damage to the ischemic tissue. This phenomenon has been termed myocardial ischemia/reperfusion injury (MI/RI) (5,6). MI/RI can lead to irreversible cell damage, ventricular dysfunction, and heart failure, which can cause sudden cardiac death (7).

Although the pathogenesis of reperfusion-induced myocardial injury is multifactorial $(8,9)$, apoptosis plays an important role in reperfusion injury $(10,11)$. Apoptosis is a highly regulated, evolutionarily conserved and energy-dependent process. It is triggered by a specific cascade of signaling pathways that ultimately lead to cell death and can result in severe organ injury $(12,13)$. Cell biology studies have demonstrated that blocking apoptosis may prevent the loss of contractile cells, minimize ischemia/reperfusion-induced cardiac injury, and, thus, retard or prevent the occurrence of heart failure (14). Therefore, the exploration of anti-apoptotic agents is a viable approach to the optimization of reperfusion strategies.

Aralia taibaiensis Z. Z. Wang \& H. C. Zheng, a member of the Araliaceae family, is a widely distributed species in the Qinba Mountains of Western China (15). The bark and root cortex are widely used in traditional folk medicine for the treatment of diabetes mellitus, hepatitis and stomach ulcers (16). In previous investigations, it was identified that total saponins, particularly triterpenoid saponins, extracted from Aralia taibaiensis (hereafter referred to as sAT) are the main pharmacologically active components of Aralia taibaiensis $(15,17)$. In addition, sAT had potent antidiabetic activity $(16,18,19)$ and anti-aging effect $(18)$, which are associated with cardiac disease, particularly ischemic disease. Thus sAT has potential protective effects against MI/RI. However, the anti-MI/RI properties of sAT have yet to be examined and the possible molecular mechanisms involved to be determined. 
The present study was therefore undertaken to investigate the anti-MI/RI activities of sAT and to elucidate the mechanisms underlying these effects in rats. The results provided important insights into the effects of sAT in cardiac disease.

\section{Materials and methods}

Materials. Dulbecco's modified Eagle's medium (DMEM) and fetal bovine serum (FBS) were purchased from Gibco-BRL (Grand Island, NY, USA). The kits used for the determination of serum lactate dehydrogenase (LDH) and creatine kinase isoenzyme-MB (CK-MB) content were obtained from Jiancheng Bioengineering Institute (Nanjing, China). MTT [3-(4,5-dimethylthiazol-2-yl)-2,5-diphenyltetrazolium bromide], TTC (2,3,5-triphenyltetrazolium chloride), Evans blue, and compound C [AMP-activated protein kinase (AMPK) inhibitor] were purchased from Sigma-Aldrich (St. Louis, MO, USA). The primary antibodies were as follows: anti-AMPK $\alpha$ (Thr172; \#2532), anti-phosphorylated AMPK $\alpha$ (p-AMPK $\alpha$ ) (Thr172; \#2531), anti-caspase-3 (\#9662), anti-Bcl-2 associated X protein (Bax; \#2772), anti-cytochrome $c$ (\#11940), anti-acetyl CoA carboxylase (ACC; \#3676), anti-p-ACC (\#11818), $\beta$-actin (\#4970), and anti-Bcl-2 (\#2870; all from Cell Signaling Technologies, Beverly, MA, USA). All these are rabbit monoclonal antibodies. The secondary antibody (anti-rabbit IgG-B; sc-53804) was purchased from Santa Cruz Biotechnology (Santa Cruz, CA, USA). The caspase-3 assay kit was purchased from Chemicon International, Inc. (Temecula, CA, USA). The fluorescent kit for Hoechst 33258 was obtained from Roche Diagnostics (Mannheim, Germany).

Plant materials. The root bark of Aralia taibaiensis Z. Z. Wang \& H.C.Zheng was collected at Mountain Taibai, Shaanxi, China, in September 2008, and botanically identified by Dr Haifeng Tang (Department of Pharmacy, Xijing Hospital, and Fourth Military Medical University, Shaanxi, China). A voucher specimen (FMMUDP-Voucher No. SAP012) was deposited in the Herbarium of the Department of Pharmacy, Xijing Hospital, and the Fourth Military Medical University, China.

Preparation of total saponins. A crude extract (total saponins) of Aralia taibaiensis (sAT) was prepared as previously described, with slight modifications (16). Dry, powdered root bark $(20 \mathrm{~g})$ was extracted three times with $80 \%(\mathrm{v} / \mathrm{v})$ ethanol (herb:ethanol, 1:10, w/v) under reflux $\left(80^{\circ} \mathrm{C}\right)$ for $60 \mathrm{~min}$. The alcohol extract was concentrated, suspended in distilled water, and then partitioned successively with chloroform (ratio 1:3, v/v) and n-butanol saturated with water (ratio 1:3, v/v, three times). The n-butanol extracts were combined and evaporated using a rotary evaporator at $60^{\circ} \mathrm{C}$ to produce a powdered residue. The yield was $0.78 \%(\mathrm{w} / \mathrm{w})$. Oleanolic acid was used as a reference standard, and the total saponins content was expressed as oleanolic acid equivalents (i.e., $459.30 \mu \mathrm{g}$ oleanolic acid equivalents/ mg extract). The content of total saponins in sAT was $>90 \%$.

Animal treatment schedule. sAT was dissolved in physiological saline $(0.9 \% \mathrm{NaCl})$ prior to use. After a 1-week-long adaptation period, the animals were randomized into five experimental groups of eight animals each as follows: sham-operated group + saline, MI/RI group + saline, MI/
RI + sAT (60 mg/kg/day, orally), MI/RI + sAT (120 mg/kg/day, orally), MI/RI + sAT (240 mg/kg/day, orally). Pretreatment with sAT or saline was initiated 7 days prior to the operation and administered once a day for 7 days.

Animal preparation for MI/RI. Experimental procedures for these animals were conducted according to the National Institutes of Health Guidelines for the Use of Laboratory Animals and were approved by the Institutional Animal Care and Use Committee of the Fourth Military Medical University. Male Sprague-Dawley rats $(250 \pm 30 \mathrm{~g} ; 10 \pm 0.5$ weeks $)$ were anesthetized with $10 \%$ chloral hydrate $(0.35 \mathrm{ml} / 100 \mathrm{~g}$ body weight) and ventilated using a positive-pressure respirator for small animals (HX-100E, Chengdu Technology \& Market Co., Chengdu, China). Electrodes were placed subcutaneously and connected to an electrocardiograph (BL-420S; Taimeng Science Technology, Ltd., Chengdu, China). During surgery, body temperature was measured and maintained at $37^{\circ} \mathrm{C}$ by placing the rats on a heating pad. After left thoracotomy, the left anterior descending coronary artery was occluded with a 6-0 silk suture. After occlusion for $30 \mathrm{~min}$, the suture was loosened, and the myocardium was reperfused for $3 \mathrm{~h}$. Sham-operated rats underwent identical surgery, but the suture was not tightened around the coronary artery. ST-segment elevation in experimental animals was assessed as a measure of myocardial ischemia as previously described (20).

After $3 \mathrm{~h}$ of reperfusion, blood samples were collected from the abdominal aorta to measure serum LDH and CK-MB levels by using an enzyme-linked immunosorbent assay (Nanjing Jiancheng Bioengineering Institute, Nanjing, China). The assay was performed according to the manufacturer's instructions. Following anesthesia, the rats were sacrificed by exsanguination (blood was drawn from the abdominal aorta) and the hearts were harvested for double-staining.

Measurement of myocardial infarct size. Myocardial infarct size was measured using a double-staining technique with $3 \%$ Evans blue and 4\% TTC adapted as previously described (21). After $6 \mathrm{~h}$ of reperfusion, the coronary artery was again occluded. To map the areas at risk for ischemia, 3\% Evans blue solution was infused through the left jugular vein. The heart was quickly excised and frozen at $-20^{\circ} \mathrm{C}$, and the ventricles were then crosssectioned into five sections. The sections were counterstained with $4 \%$ TTC (in phosphate buffer, $\mathrm{pH} 7.8$ ) for $15 \mathrm{~min}$ at $37^{\circ} \mathrm{C}$ and photographed after overnight storage in $4 \%$ paraformaldehyde. The infarct size was presented as the left ventricular infarct area as a percentage of the ischemic area at risk.

TUNEL staining for apoptosis in vivo. To examine cardiac myocyte apoptosis, samples of tissue from the ischemic zones were fixed in $4 \%$ paraformaldehyde, embedded in paraffin, and cut into 5-mm transverse sections. The terminal deoxynucleotidyl transferase-mediated dUTP nick end-labeling (TUNEL) assay was carried out using an apoptosis detection kit (Roche Applied Science, Mannheim, Germany) according to the manufacturer's instructions. 4',6-Diamidino-2-phenylindole (DAPI) was used as a counterstain. The apoptotic cells were analyzed by laser-scanning confocal microscopy (SP5-FCS; Leica Microsystems, Wetzlar, Germany). The number of TUNELpositive nuclei was calculated by ImageJ software $(\mathrm{NIH}$, 
Bethesda, MD, USA). Quantitative analysis was carried out using the following formula: percentage of TUNEL-positive cells $=$ TUNEL-positive cells/total cells.

$H 9 c 2$ cell culture and treatment. Rat $\mathrm{H} 9 \mathrm{c} 2$ cardiomyocytes were obtained from the American Type Culture Collection (ATCC; Manassas, VA, USA). The cells were cultured in DMEM with $10 \%$ fetal bovine serum (FBS), $100 \mathrm{U} / \mathrm{ml}$ penicillin, and $100 \mathrm{mg} / \mathrm{ml}$ streptomycin and maintained in a humidified atmosphere of $5 \% \mathrm{CO}_{2}$ at $37^{\circ} \mathrm{C}$. The medium was replaced every 2-3 days, and the cells were subcultured at $80-90 \%$ confluence by detaching with $0.05 \%$ trypsin-ethylenediaminetetraacetic acid and reseeding.

Anoxia/reoxygenation $(A / R)$ injury model. To mimic ischemic injury in vitro, ischemia and reperfusion were performed in rat H9c2 cells based on a previously described method (22). At $80 \%$ confluence, the cells were incubated in an 'ischemic buffer' for $2 \mathrm{~h}$ at $37^{\circ} \mathrm{C}$ in a hypoxic chamber (previously bubbled with $95 \% \mathrm{~N}_{2}$ and $5 \% \mathrm{CO}_{2}$ ). The buffer contained $137 \mathrm{mM} \mathrm{NaCl}$, $12 \mathrm{mM} \mathrm{KCl}, 0.49 \mathrm{mM} \mathrm{MgCl}_{2}, 0.9 \mathrm{mM} \mathrm{CaCl}_{2} \cdot 2 \mathrm{H}_{2} \mathrm{O}, 4 \mathrm{mM}$ HEPES, and $20 \mathrm{mM}$ sodium lactate (pH 6.2). For the A/R studies, the medium was replaced by maintenance medium (DMEM with 2\% FBS) during the re-oxygenation period.

Cell viability. Cell viability was determined colorimetrically using the MTT assay. Cells at the exponential phase were seeded at $1 \times 10^{4}$ cells/well in 96-well plates. After different treatments, $20 \mu \mathrm{l}$ of $5 \mathrm{mg} / \mathrm{ml} \mathrm{MTT} \mathrm{solution} \mathrm{was} \mathrm{added} \mathrm{to}$ each well $(0.5 \mathrm{mg} / \mathrm{ml}$ final concentration), and the plates were incubated for $4 \mathrm{~h}$ at $37^{\circ} \mathrm{C}$. The supernatant was removed, the formazan crystals were dissolved in $150 \mu \mathrm{l}$ DMSO, and the optical density at $490 \mathrm{~nm}$ was read on a microplate reader (Tecan, Mannedorf, Switzerland). The cell survival ratio was expressed as a percentage of the control.

Determination of $\mathrm{LDH}$ and $C K-M B$ release in culture medium. The cell culture medium was collected after the $\mathrm{A} / \mathrm{R}$ procedure. The concentrations of $\mathrm{LDH}$ and CK-MB were determined by a colorimetric method using commercial kits (Nanjing Jiancheng Bioengineering Institute) according to the manufacturer's protocol. The results were reported as U/l.

Hoechst 33258 staining assay. Briefly, H9c2 cardiomyocytes were washed with ice-cold PBS, fixed in $10 \%$ neutral buffered formalin for $10 \mathrm{~min}$ at room temperature, and washed again in ice-cold PBS. The cardiomyocytes were then exposed to Hoechst $33258(2 \mu \mathrm{g} / \mathrm{ml}$ in PBS $)$ and incubated for $20 \mathrm{~min}$ at room temperature. The cells were then washed three times in PBS and examined under a fluorescence microscope with an appropriate filter. Images were captured at a magnification of x200 using an Olympus microscope (1X71; Olympus Corp., Tokyo, Japan).

Measurement of caspase-3 activity. Caspase-3 activity was determined in cytosolic protein extracts using a colorimetric activity assay kit (Beyotime Institute of Biotechnology, Haimen, China) according to the manufacturer's protocol. Specifically, after determining the protein concentration, the supernatant was incubated with the caspase-3 substrate
(Ac-DEVD-pNA) on a 96-well-plate. The activity of caspase-3 was detected using a microplate reader (Tecan) at $405 \mathrm{~nm}$.

Protein extraction and western blot analysis. H9c2 cells cultured in 6 -well plates $\left(3 \times 10^{5} /\right.$ well) were washed twice with ice-cold PBS and lysed via incubation on ice for $30 \mathrm{~min}$ with lysis buffer ( $\mathrm{pH} 7.5$ ) containing $20 \mathrm{mM}$ Tris- $\mathrm{HCl}, 120 \mathrm{mM}$ $\mathrm{NaCl}, 1.0 \%$ Triton $\mathrm{X}-100,10 \%$ glycerol, $2 \mathrm{mM}$ ethylenediaminetetraacetic acid, and protease inhibitor cocktail (Roche $\mathrm{GmbH})$. Following centrifugation of the cell lysates at $4^{\circ} \mathrm{C}$ for $20 \mathrm{~min}$ at $10,000 \mathrm{x} \mathrm{g}$, the supernatants (total cell extracts) were frozen and stored at $-80^{\circ} \mathrm{C}$. The protein concentration of each sample was determined using a bicinchoninic acid assay protein assay kit (Pierce Chemical, Rockford, IL, USA).

For the western blot analysis, equal amounts of cytosolic protein lysates were separated by sodium dodecyl sulfate-polyacrylamide gel electrophoresis on 10-15\% gels. The gels were transferred onto polyvinylidene fluoride membranes (PVDF; Bio-Rad, Hercules, CA, USA), blocked for $30 \mathrm{~min}$ at $37^{\circ} \mathrm{C}$ with $5 \%$ non-fat dry milk, and incubated with the primary antibodies (1:1,000 dilution) overnight at $4^{\circ} \mathrm{C}$. After three washes with Tris-buffered saline plus Tween-20, the membranes were incubated with secondary antibodies (1:200 dilution) in Trisbuffered saline plus Tween-20 for $30 \mathrm{~min}$ at $37^{\circ} \mathrm{C}$ and washed as described above. Subsequently, the blots were developed by enhanced chemiluminescence according to the manufacturer's instructions.

Statistical analysis. Data were presented as mean \pm standard deviation. The statistical significance of differences between the means were determined using SPSS 19 software (SPSS, Inc., Chicago, IL, USA) for Windows using one-way analysis of variance followed by the Bonferroni post hoc test or Student's $\mathrm{t}$-test, as appropriate. Differences with P-values of $<0.05$ were considered statistically significant.

\section{Results}

sAT protected rats against apoptosis induced by $M I / R I$. Myocardial infarct size, plasma CK, and plasma LDH were the primary indicators used to assess myocardial injury after MI/RI. Representative areas at risk and infarct images are shown in Fig. 1A and B. No myocardial infarction was observed in hearts from the sham-operated group. However, significant infarction was observed in rats in the MI/RI group compared to rats in the sham group $[50.8 \pm 1.5 \%$ (infarct area as a percentage of area at risk), $\mathrm{P}<0.01]$. Treatment with 120 and $240 \mathrm{mg} / \mathrm{kg}$ sAT significantly decreased infarct size compared to the MI/RI-group $(39.6 \pm 3.3 \%, \mathrm{P}<0.05$ and $22.0 \pm 3.0 \%$, $\mathrm{P}<0.01$, respectively). There was no significant difference in the area at risk between any of the groups.

Injury to the cardiomyocytes was determined by measuring the levels of LDH and CK-MB in the serum at the end of reperfusion (Fig. 1C and D). MI/RI significantly increased LDH and CK-MB levels compared to those in the sham group $(1185.5 \pm 83.7 \%, \mathrm{P}<0.01$ and $3523.4 \pm 326.9 \%, \mathrm{P}<0.01$, respectively), while 120 and $240 \mathrm{mg} / \mathrm{kg}$ sAT markedly reduced the levels of LDH and CK-MB after MI/RI $(\mathrm{P}<0.01)$.

Consistent with these results, the MI/RI group exhibited a significant increase in TUNEL-positive cells compared with 
A

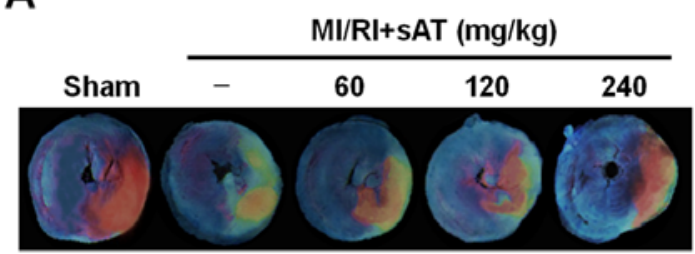

B

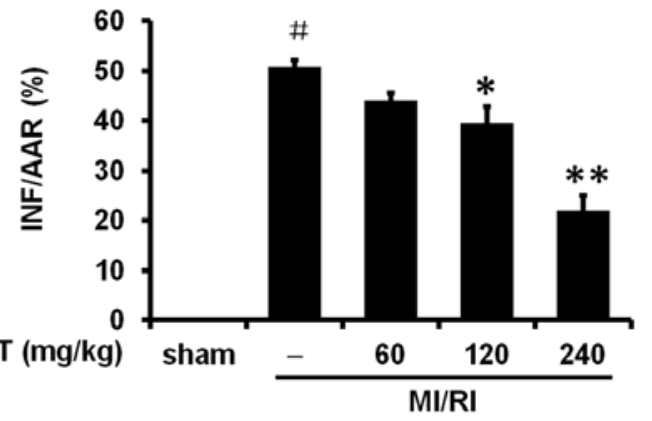

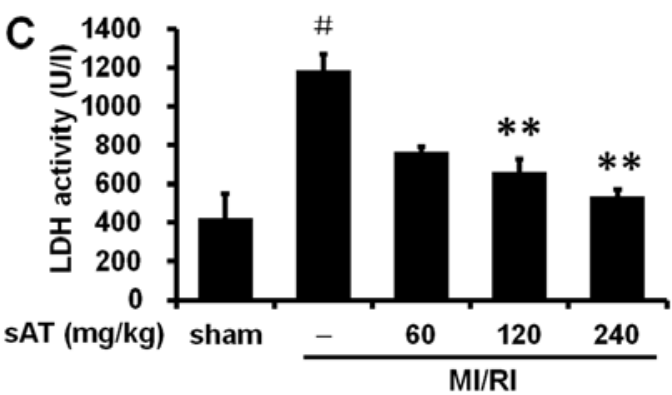

D

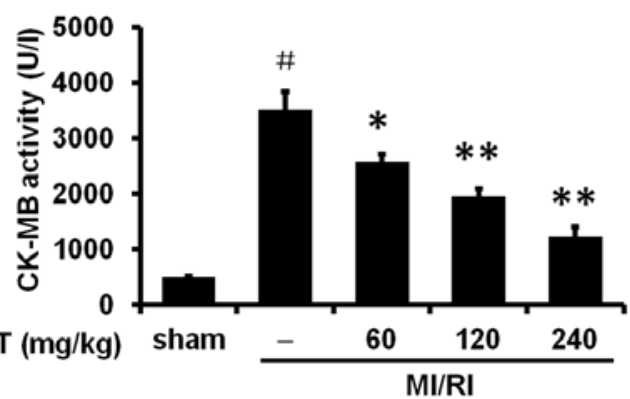

Figure 1. Effects of total saponins extracted from Aralia taibaiensis (sAT) on myocardial damage in rats subjected to myocardial ischemia/reperfusion injury (MI/RI). (A) Representative photomicrographs of heart sections stained by Evans blue (indicating non-ischemic/reperfused areas) and 2,3,5-triphenyltetrazolium chloride (TTC; indicating the ischemic area) in different treatment groups. (B) Effect of sAT on myocardial infarct size (INF) expressed as a percentage of the total ischemic-reperfused area-at-risk (AAR). (C) Effect of sAT on the activity of lactate dehydrogenase (LDH) in the serum of rats subjected to MI/RI. (D) Effect of sAT on the activity of creatine kinase isoenzyme MB (CK-MB) in the serum of rats subjected to MI/RI. Data are shown as mean \pm standard deviation of three independent experiments. ${ }^{*} \mathrm{P}<0.01$ vs. sham group; ${ }^{*} \mathrm{P}<0.05,{ }^{* *} \mathrm{P}<0.01$ vs. MI/RI group.
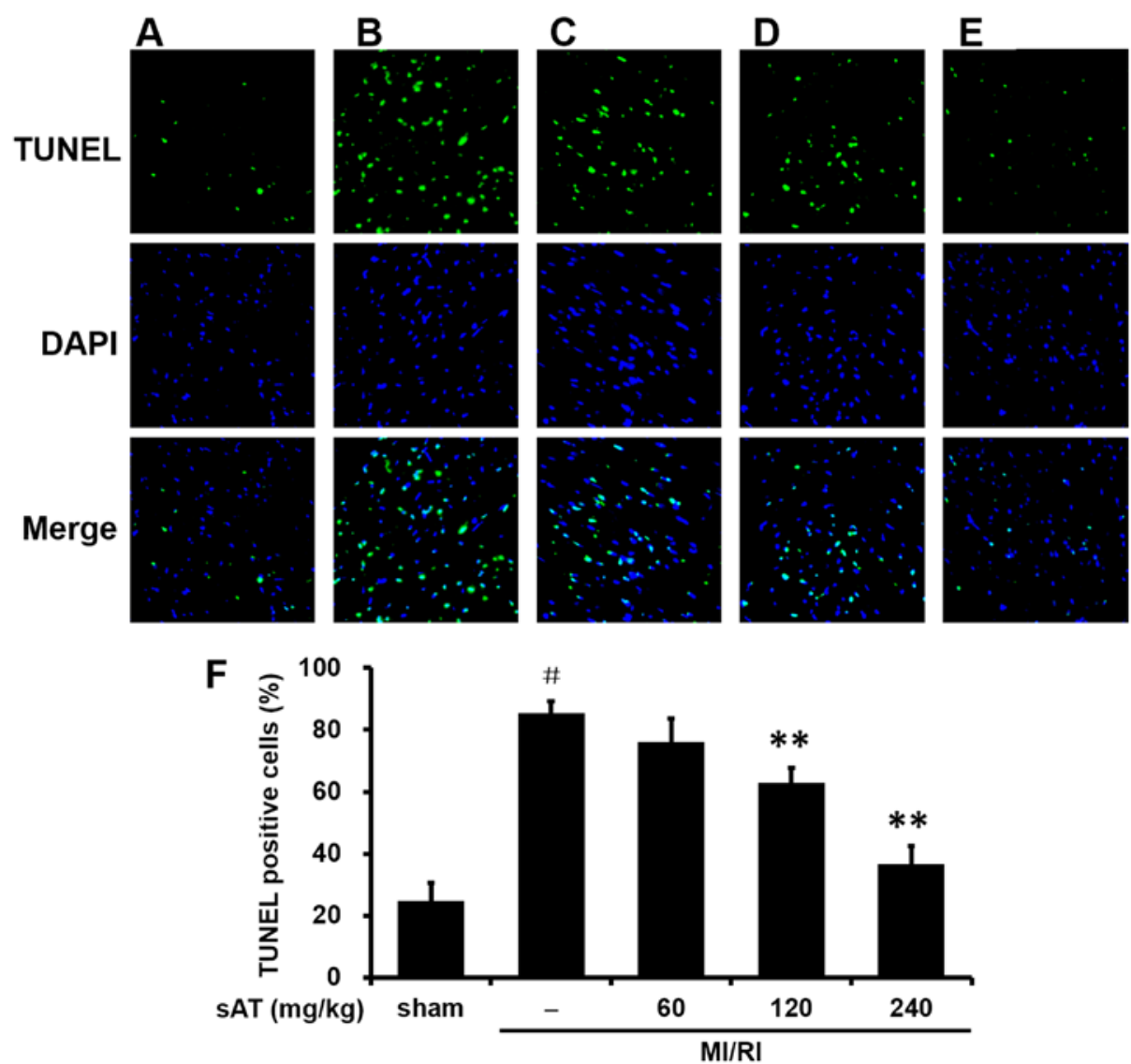

Figure 2. Terminal deoxynucleotidyl transferase-mediated dUTP nick end-labeling (TUNEL) staining and morphological analysis of apoptotic damage in isolated rat hearts treated with total saponins extracted from Aralia taibaiensis (sAT) followed by myocardial ischemia/reperfusion injury (MI/RI). (A) Sham + saline (vehicle), (B) MI/RI + saline, (C) MI/RI + sAT $60 \mathrm{mg} / \mathrm{kg}$, (D) MI/RI + sAT $120 \mathrm{mg} / \mathrm{kg}$, (E) MI/RI + sAT $240 \mathrm{mg} / \mathrm{kg}$, (F) quantitative analysis of TUNEL-positive cells. Data are shown as mean \pm standard deviation of three independent experiments. ${ }^{*} \mathrm{P}<0.01$ vs. sham group; ${ }^{*} \mathrm{P}<0.05$, ${ }^{* * *} \mathrm{P}<0.01$ vs. MI/RI group. 

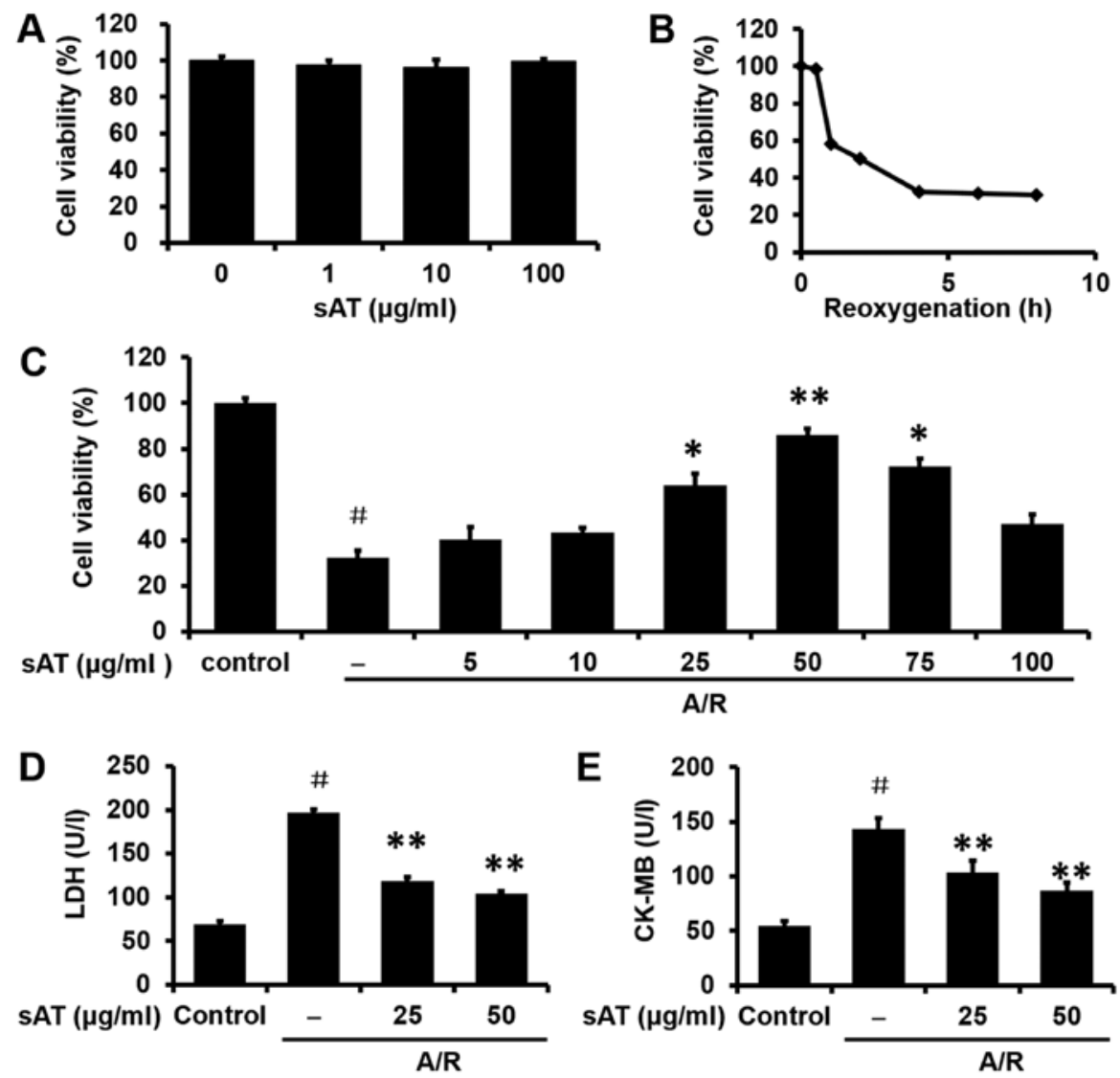

Figure 3. Total saponins extracted from Aralia taibaiensis (sAT) protected H9c2 cells from anoxia/re-oxygenation (A/R)-induced damage measured by the 3-(4,5-dimethylthiazol-2-yl)-2,5-diphenyl-tetrazolium bromide (MTT) assay. (A) Toxic effects of sAT (1-100 $\mu \mathrm{g} / \mathrm{ml})$ in H9c2 cells after 24-h incubation. (B) Time-dependent effects of re-oxygenation on cell viability. (C) Protective effects of different concentrations of sAT (5-100 $\mu \mathrm{g} / \mathrm{ml})$ on anoxia-induced cytotoxicity in H9c2 cells. (D) The effect of sAT on the activity of lactate dehydrogenase (LDH) in the culture medium. (E) The effect of sAT on the activity of creatine kinase isoenzyme MB (CK-MB) in the culture medium. All data are shown as mean \pm standard deviation of three independent experiments. ${ }^{\#} \mathrm{P}<0.01$ vs. the control group; ${ }^{*} \mathrm{P}<0.05,{ }^{* *} \mathrm{P}<0.01$ vs. the $\mathrm{A} / \mathrm{R}$ group.

the sham-group $(85.5 \pm 3.7 \%, \mathrm{P}<0.01)$ (Fig. 2). Pretreatment with 120 or $240 \mathrm{mg} / \mathrm{kg}$ sAT substantially reduced the number of TUNEL-positive cells $(62.9 \pm 4.8 \%, \mathrm{P}<0.01$ and $36.6 \pm 5.7 \%$, $\mathrm{P}<0.01$, respectively). Taken together, these data suggested that sAT decreased post-MI/RI myocardial apoptosis in vivo.

sAT protects $H 9 c 2$ cells following $A / R$ injury challenge. The toxicity of sAT was examined in cultured H9c2 cells incubated with sAT $(1-100 \mu \mathrm{g} / \mathrm{ml})$ for $24 \mathrm{~h}$. As shown in Fig. 3A, sAT did not alter cell viability at concentrations reaching $100 \mu \mathrm{g} / \mathrm{ml}$. The time course of the loss of cell viability following A/R injury was also assessed using MTT assays (Fig. 3B). Based on these data, $4 \mathrm{~h}$ was selected as the re-oxygenation time to measure the markers of cell injury. Although the A/R treatment significantly reduced cell survival, pretreatment with sAT markedly increased survival in a concentration-dependent manner. The $50 \mu \mathrm{g} / \mathrm{ml}$ concentration was selected for subsequent investigation (Fig. 3C). LDH and CK-MB activities were measured in the supernatants of culture media. An increase in LDH and CK-MB levels was observed in the $\mathrm{A} / \mathrm{R}$ group compared to the corresponding levels in the control group $(197.2 \pm 3.4 \%, \mathrm{P}<0.01$ and $143.5 \pm 9.7 \%, \mathrm{P}<0.01$, respectively; Fig. $3 \mathrm{D}$ and $\mathrm{E}$ ). The levels of LDH and CK-MB were both decreased by sAT as compared to the corresponding levels in the $\mathrm{A} / \mathrm{R}$ group $(\mathrm{P}<0.01)$.
SAT protects H9C2 cardiomyocytes from apoptosis induced by $A / R$ injury. Subsequent to A/R injury, cardiomyocytes exhibited typical characteristics of apoptosis, including shrinkage of the nuclei, chromatin condensation, and the appearance of apoptotic bodies. However, the number of cells with nuclear condensation and fragmentation was significantly decreased in cardiomyocytes incubated with sAT for $24 \mathrm{~h}$ following A/R injury (Fig. 4A). Quantitative analysis results are shown in Fig. 4B. Similar data were obtained from observation of the effects of sAT on caspase-3 activity (Fig. 4C) and protein expression of cleaved caspase-3 (Fig. 4D) and cytochrome $c$ (Cyto-c; Fig. 4E). The expression of Bax, a proapoptotic protein, was markedly increased after $\mathrm{A} / \mathrm{R}$ injury, but was decreased in cells treated with sAT compared to A/R alone (Fig. 4F). At the same time, the expression of Bcl-2, an anti-apoptotic protein, decreased in the $\mathrm{A} / \mathrm{R}$ group and increased in the sAT-treated groups. The Bcl-2/Bax ratio was also decreased in $\mathrm{H} 9 \mathrm{c} 2$ cells following $\mathrm{A} / \mathrm{R}$ injury compared to normoxic control cells $(0.54 \pm 0.12 \%, \mathrm{P}<0.01)$, and this decrease was reversed by treatment with sAT $(2.27 \pm 0.31 \%$, $\mathrm{P}<0.01$ and $2.78 \pm 0.17 \%, \mathrm{P}<0.01$, respectively).

AMPK activation is involved in the cardioprotective effects of $s A T$. To investigate the possible mechanisms involved in 
A

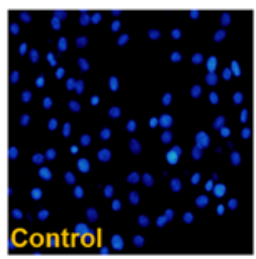

B

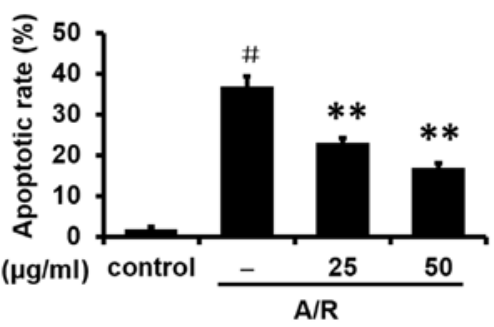

D

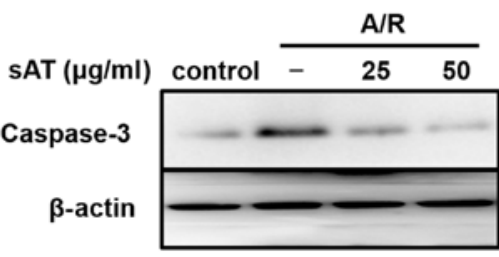

E
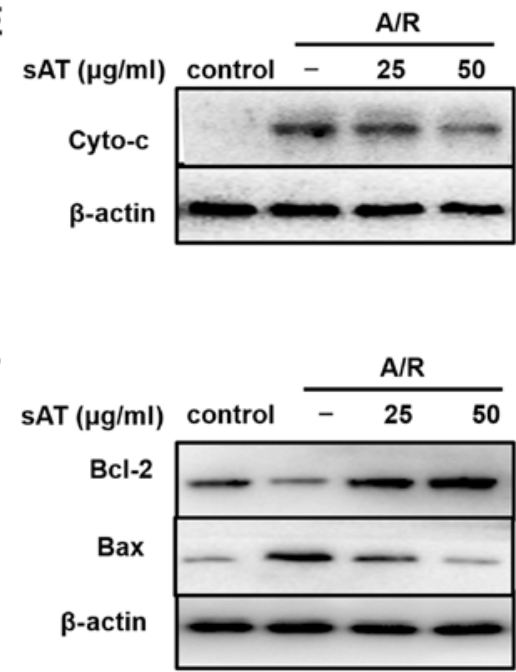
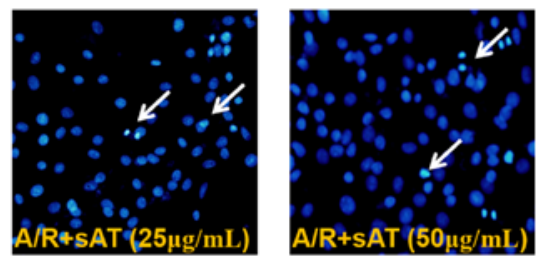

C
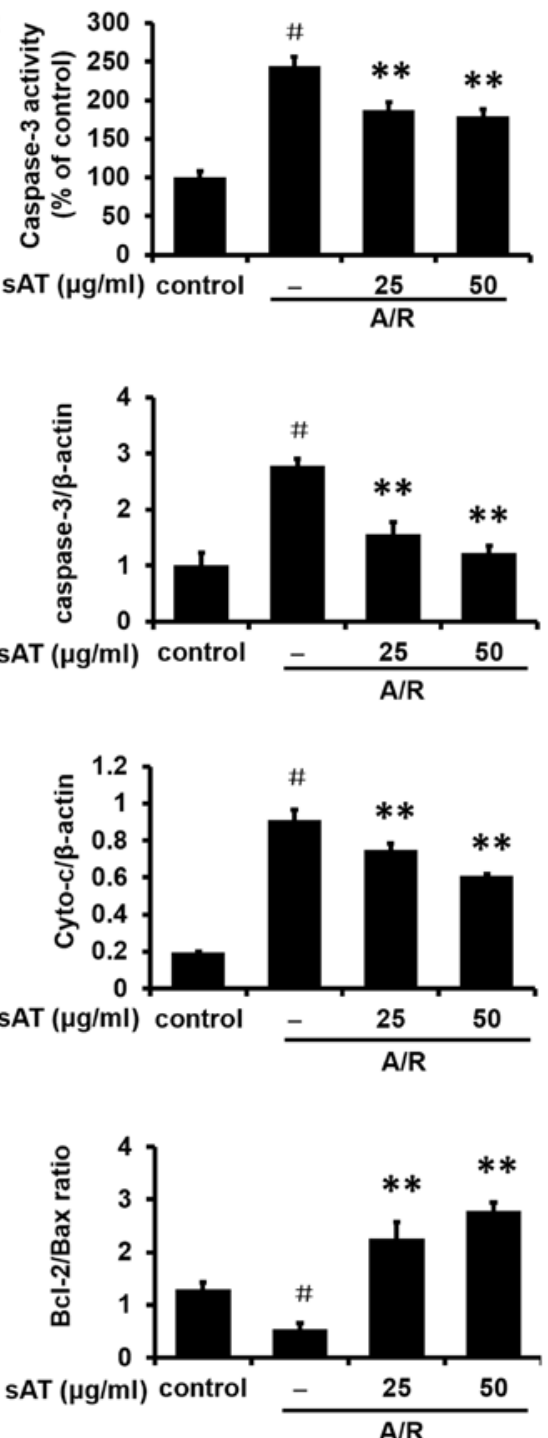

Figure 4. Total saponins extracted from Aralia taibaiensis (sAT) protected H9c2 cells from anoxia/re-oxygenation (A/R)-induced apoptosis. (A) Anti-apoptotic effects on cardiomyocytes following A/R injury were confirmed by Hoechst 33258 staining. Quantitative analysis of apoptosis is shown in (B). (C) Caspase-3 activity was determined in cytosolic extracts from cells pretreated with sAT for $24 \mathrm{~h}$ prior to A/R injury. Proteins were extracted and subjected to western blot analysis. The expression levels of the cleaved form of caspase-3, cytochrome $c$ (Cyto-c), Bcl-2-associated X protein (Bax), and Bcl-2 proteins are shown in (D), (E) and $(\mathrm{F})$, respectively. Data are shown as mean \pm standard deviation of three independent experiments. ${ }^{\#} \mathrm{P}<0.01 \mathrm{vs}$. the control group; ${ }^{*} \mathrm{P}<0.05,{ }^{* *} \mathrm{P}<0.01$ vs. the A/R group.

the cardioprotective effects of sAT, the expression levels of proteins associated with the AMPK pathway were determined by western blot analysis. As shown in Fig. 5A, the phosphorylation of AMPK was investigated in $\mathrm{H} 9 \mathrm{c} 2$ cardiomyocytes exposed to A/R with different re-oxygenation times (10, 20, 40, 60 and $120 \mathrm{~min}$ ). Since the highest level of p-AMPK (relative to total AMPK) was reached at $40 \mathrm{~min}$, this time point was selected for subsequent mechanistic studies. Enhancement of AMPK and ACC phosphorylation was observed in the cells incubated with sAT after A/R injury compared with the control group (Fig. 5B and C).
$A M P K$ is required for the anti-apoptotic effects of $S A T$ in $H 9 c 2$ cells. To investigate whether the AMPK pathway was required for the protective effect of $\mathrm{SAT}$ in cardiomyocytes subjected to A/R injury, cells were treated with the AMPK inhibitor, compound $\mathrm{C}(0.5 \mathrm{mM})$ for $1 \mathrm{~h}$ prior to incubation with sAT. Enhancement of AMPK phosphorylation (Fig. 6A) and ACC phosphorylation (Fig. 6B) by sAT was eradicated by treatment with compound $\mathrm{C}$. In addition, the expression of Bax and Bcl-2 and the activity of caspase- 3 were measured following the incubation of cells under $\mathrm{A} / \mathrm{R}$ with or without compound $\mathrm{C}$ in the presence of sAT. As shown in Fig. 6C 
A

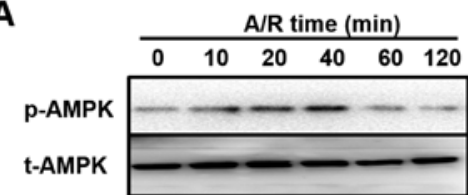

B

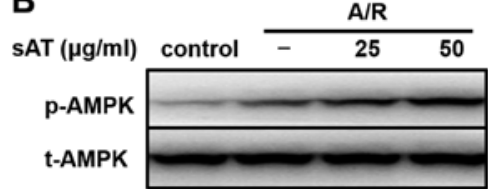

C

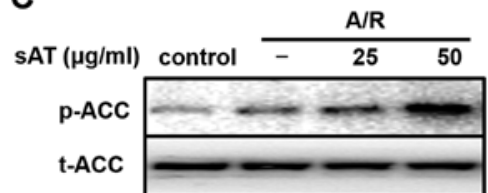

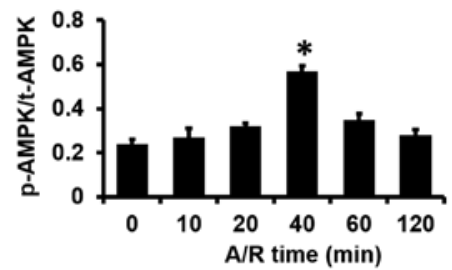

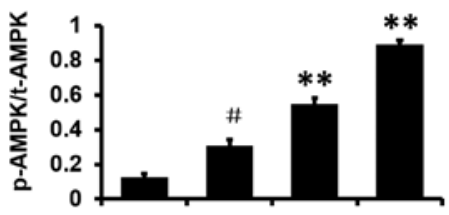

SAT $(\mu \mathrm{g} / \mathrm{ml})$ control
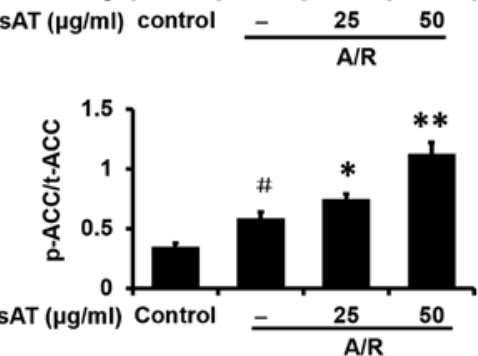

Figure 5. Total saponins extracted from Aralia taibaiensis (sAT) promote AMP-activated protein kinase (AMPK) activation. Western blots were performed with cytosolic extracts from the cells treated with sAT or vehicle. The samples were immunoblotted and probed with antibodies. Representative western blots are provided. (A) The time course of AMPK phosphorylation was determined under normoxic conditions and subsequent to A/R injury. (B) Effects of sAT on AMPK phosphorylation. (C) Formation of phosphorylated acetyl CoA carboxylase (p-ACC) in the presence of sAT. Data are shown as mean \pm standard deviation of three independent experiments. ${ }^{\#} \mathrm{P}<0.01$ vs. the control group; ${ }^{*} \mathrm{P}<0.05,{ }^{* *} \mathrm{P}<0.01$ vs. the $\mathrm{A} / \mathrm{R}$ group.

\section{A}
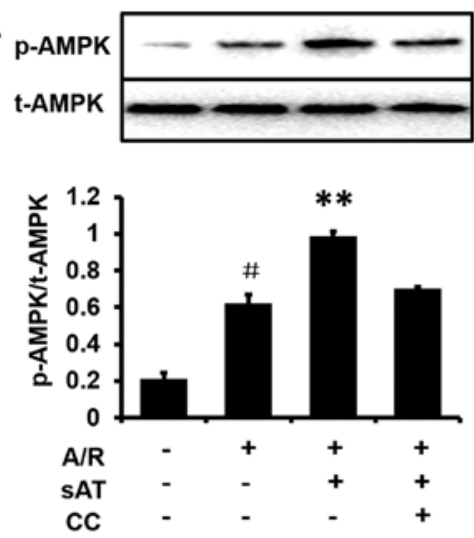

C
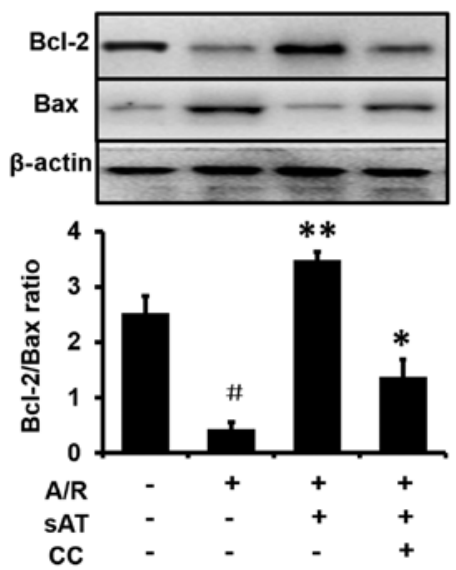

B
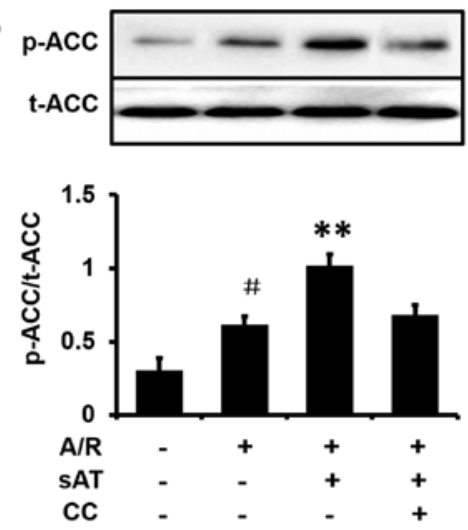

D

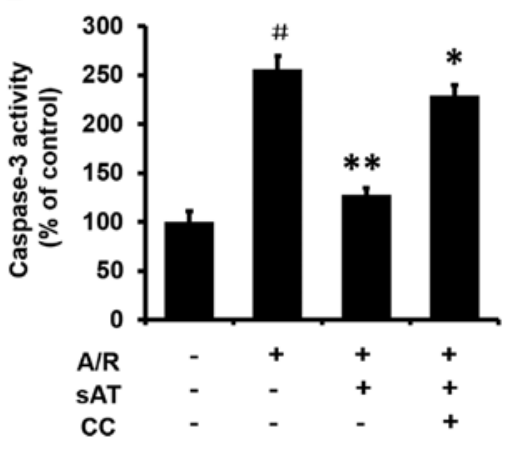

Figure 6. Total saponins extracted from Aralia taibaiensis (sAT) require AMP-activated protein kinase (AMPK) activation for their anti-apoptotic effects. (A) Phosphorylated AMPK (p-AMPK) and total AMPK (t-AMPK) were measured by western blot analysis using anti-p-AMPK and anti-AMPK antibodies. The protein signals were quantified by densitometry, and the graph shows their relative levels. (B) Phosphorylated acetyl CoA carboxylase (p-ACC) and total ACC (t-ACC) were determined, and densitometric analysis of the bands is shown in the graph. (C) Levels of Bcl-2 and Bax were analyzed in the presence or absence of the AMPK inhibitor, compound C (CC), and/or sAT (50 $\mu \mathrm{g} / \mathrm{ml})$. $\beta$-actin was used as a loading control. (D) Densitometry of caspase-3 activity levels in the presence or absence of the AMPK inhibitor, compound C (CC) and/or sAT $(50 \mu \mathrm{g} / \mathrm{ml})$. Data are shown as mean \pm standard deviation of three independent experiments. ${ }^{*} \mathrm{P}<0.01$ vs. the control group; ${ }^{*} \mathrm{P}<0.05,{ }^{* *} \mathrm{P}<0.01$ vs. the $\mathrm{A} / \mathrm{R}$ group. 
and D, compared with the sAT-treated group, treatment with sAT plus compound $\mathrm{C}$ markedly inhibited the increase in the $\mathrm{Bcl}-2 / \mathrm{Bax}$ ratio $(1.38 \pm 0.31 \%, \mathrm{P}<0.05)$ and the reduction in caspase-3 activity $(229.0 \pm 10.6 \%, \mathrm{P}<0.05)$.

\section{Discussion}

In the present study, we investigated the potential protective effects of sAT against MI/RI in rats. We found that sAT reduced the damage from $\mathrm{MI} / \mathrm{RI}$ to the rat heart after left anterior descending coronary artery occlusion. Rats treated with sAT had a smaller infarct size compared with the MI/RI rats treated with vehicle. Biochemical measures of cardiac damage (serum LDH and CK-MB) also supported the protective effects of sAT. Furthermore, the suppression of cardiomyocyte apoptosis and activation of the AMPK pathway were observed in cardiomyocytes in vitro and may contribute to the cardioprotective effects of sAT.

Acute ischemic injury resulting from coronary artery disease leads to myocardial cell death, most likely due to ATP depletion, increased calcium load, acidosis and oxidative stress $(23,24)$. In reperfusion injury, the initiating event is the interruption and subsequent re-establishment of blood supply to the tissue, which leads to extensive secondary cardiac injury and tissue damage (25). In the past 20 years, preconditioning and postconditioning treatments have been applied to reduce reperfusion injury (26). However, the use of these treatments in humans to reduce infarct size and improve catheterization outcomes is impractical. Consequently, basic and applied research should be directed at the identification of pharmacological agents that can reproducibly mimic these techniques and outcomes in humans.

Findings of previous studies have shown that sAT exerts antidiabetic and anti-aging effects $(16,18)$. Diabetes and aging are risk factors for developing cardiovascular diseases, particularly ischemic heart disease. In addition, it has been previously demonstrated that Aralia species contain some ginseng-like triterpenoid saponins. The effect produced by saponins from Aralia species is similar to the effects of panaxosides from ginseng. These effects may contribute to the ability of Aralia plants to increase energy, strengthen the body, and improve the response of the body to hypoxia with regard to the cardiovascular system (27-30). In the present study, we identified that sAT exhibited anti-MI/RI effects. Specifically, 120 and $240 \mathrm{mg} / \mathrm{kg}$ sAT significantly reduced infarct size in rat hearts. sAT also caused increases in serum LDH and CK-MB, two cytosolic enzymes that serve as diagnostic markers of ischemia injury to the myocardium and leak out from damaged myocardial tissues to the blood stream when the cell membrane becomes permeable or ruptures. The two phenomena demonstrated that sAT may reduce and delay acute MI/RI.

Apoptosis can lead to protein cleavage, DNA breakdown and nuclear shrinkage (31). We found that pretreatment with sAT minimized cardiac injury by preventing apoptosis induced by MI/RI in vivo and in vitro. Notably, sAT pretreatment markedly decreased the number of TUNEL-positive cells and prevented morphological changes in the nucleus, as observed by Hoechst staining, when compared with the vehicle-treated group. Thus, we concluded that sAT had an anti-apoptotic role in the prevention of MI/RI.
Mitochondrial dysfunction is a major cause of cardiomyocyte death induced by MI/RI (32). ATP generation is the most important function of mitochondria, particularly in the heart. However, mitochondria also regulate apoptosis (33) through various pathways, such as regulation of the activity of the Bcl-2 family, inhibition of cytochrome $c$ release and inhibition of caspase activation. Thus, we examined the mechanism through which sAT protected against apoptosis in cardiomyocytes. A discussion of each of these pathways (i.e., regulation of Bcl-2 proteins, inhibition of Cyto-c release, and suppression of caspase activation) is provided below.

The Bcl-2 family, key regulators of apoptosis, comprises cell death promoters, such as Bax and Bad, and cell death inhibitors, including Bcl-2 and Bcl-x (34). High Bax/Bcl-2 ratios are associated with apoptotic activation. The results on early apoptotic signaling following A/R showed that SAT impaired the accumulation of Bax and upregulated the levels of Bcl-2 protein. Thus, the Bcl-2/Bax ratio increased following treatment with sAT. Therefore, Bcl-2 and Bax may be involved in mediating the anti-apoptotic effects of sAT after A/R injury.

Cyto-c is a pro-apoptotic signaling molecule localized within the mitochondrial cristae. Its release from the mitochondria into the cytosol is a critical event in apoptosis (35). In the present study, pretreatment with sAT significantly suppressed the release of Cyto-c from the mitochondria into the cytosol induced by $\mathrm{A} / \mathrm{R}$ injury. Of note, the release of Cyto-c from mitochondria consequently activated caspase-3, which plays a pivotal role in apoptosis (36). sAT markedly suppressed the activity and expression of caspase-3, while A/R increased the activity and expression of this critical apoptotic regulator. Thus, the protective role of sAT may be mediated by inhibition of the release of Cyto-c and suppression of the activity of caspase-3.

AMPK is a serine/threonine kinase that senses energy levels within the cell, and together with its downstream effector, ACC, coordinates metabolic responses to maintain energy homeostasis (37). Animals deficient in AMPK exhibit increased cardiac hypertrophy, accelerated heart failure and increased infarct sizes following coronary artery ligation (38). The classic function of AMPK is to maintain energy homeostasis by modulating metabolic pathways $(39,40)$. However, it has been demonstrated that AMPK may protect against reperfusion injury by regulating cell survival and limiting apoptosis rather than through its metabolic actions (41). In the present study, we observed a significant increase in the phosphorylation of AMPK and its downstream target, ACC, during reperfusion. This finding indicated that under stress conditions, sAT activated AMPK to increase ATP production in order to maintain contractile function and cell homeostasis. In addition, when the AMPK inhibitor, compound C, was administered, the activation of AMPK and ACC was suppressed. The anti-apoptotic effects of sAT were also decreased by compound $\mathrm{C}$. These results showed that pretreatment with sAT conferred resistance to A/R injury-induced apoptosis by activating the AMPK pathway in vitro. However, further studies are needed to elucidate the underlying mechanism by using a more specific animal model. The involvement of other signaling pathways in the cardioprotective effects of sAT (different from AMPK pathway) must also be addressed. In this manner, we could powerfully assess the different curative effects between sAT and positive control. 
In summary, the results of the present study have demonstrated that sAT pretreatment may reduce myocardial injury in vitro and in vivo. The protective effects of sAT may be due, in part, to the suppression of apoptosis and promotion of survival via the AMPK pathway. Ongoing investigations of the effects of sAT, including the active components and pharmacological parameters, may lead to novel therapeutic strategies for the treatment of myocardial ischemia and reperfusion injury.

\section{Acknowledgements}

The present study was supported by the National Natural Science Foundation of China (grant nos. 81470174 and 81001673).

\section{References}

1. Go AS, Mozaffarian D, Roger VL, Benjamin EJ, Berry JD, Blaha MJ, Dai S, Ford ES, Fox CS, Franco S, et al; American Heart Association Statistics Committee and Stroke Statistics Subcommittee: Executive summary: heart disease and stroke statistics--2014 update: a report from the American Heart Association. Circulation 129: 399-410, 2014.

2. Fox KA, Steg PG, Eagle KA, Goodman SG, Anderson FA Jr, Granger CB, Flather MD, Budaj A, Quill A, Gore JM, et al, GRACE Investigators: Decline in rates of death and heart failure in acute coronary syndromes, 1999-2006. JAMA 297: 1892-1900, 2007.

3. Lange RA and Hillis LD: Immediate angioplasty for acute myocardial infarction. N Engl J Med 328: 726-728, 1993.

4. Simoons ML and Windecker S: Controversies in cardiovascular medicine: Chronic stable coronary artery disease: drugs vs revascularization. Eur Heart J 31: 530-541, 2010.

5. Braunwald E and Kloner RA: Myocardial reperfusion: A doubleedged sword? J Clin Invest 76: 1713-1719, 1985.

6. Buja LM: Myocardial ischemia and reperfusion injury. Cardiovasc Pathol 14: 170-175, 2005.

7. Yellon DM and Hausenloy DJ: Myocardial reperfusion injury. N Engl J Med 357: 1121-1135, 2007.

8. Moens AL, Claeys MJ, Timmermans JP and Vrints CJ: Myocardial ischemia/reperfusion-injury, a clinical view on a complex pathophysiological process. Int J Cardiol 100: 179-190, 2005

9. Mozaffari MS, Liu JY, Abebe W and Baban B: Mechanisms of load dependency of myocardial ischemia reperfusion injury. Am J Cardiovasc Dis 3: 180-196, 2013.

10. Eefting F, Rensing B, Wigman J, Pannekoek WJ, Liu WM, Cramer MJ, Lips DJ and Doevendans PA: Role of apoptosis in reperfusion injury. Cardiovasc Res 61: 414-426, 2004.

11. MacLellan WR and Schneider MD: Death by design. Programmed cell death in cardiovascular biology and disease. Circ Res 81: 137-144, 1997.

12. Abbate A, Bussani R, Amin MS, Vetrovec GW and Baldi A: Acute myocardial infarction and heart failure: Role of apoptosis. Int J Biochem Cell Biol 38: 1834-1840, 2006.

13. Fliss $\mathrm{H}$ and Gattinger D: Apoptosis in ischemic and reperfused rat myocardium. Circ Res 79: 949-956, 1996.

14. Gill C, Mestril R and Samali A: Losing heart: The role of apoptosis in heart disease--a novel therapeutic target? FASEB J 16: 135-146, 2002

15. Tang HF, Yi YH, Wang ZZ, Hu WJ and Li YQ: Studies on the triterpenoid saponins of the root bark of Aralia taibaiensis. Yao Xue Xue Bao 31: 517-523, 1996 (In Chinese).

16. Xi M, Hai C, Tang H, Wen A, Chen H, Liu R, Liang X and Chen M: Antioxidant and antiglycation properties of triterpenoid saponins from Aralia taibaiensis traditionally used for treating diabetes mellitus. Redox Rep 15: 20-28, 2010.

17. Tang HF, Yi YH, Wang ZZ, Jiang YP and Li YQ: Oleanolic acid saponins from the root bark of Aralia taibaiensis. Yao Xue Xue Bao 32: 685-690, 1997 (In Chinese).

18. Li YN, Guo Y, Xi MM, Yang P, Zhou XY, Yin S, Hai CX, Li JG and Qin XJ: Saponins from Aralia taibaiensis attenuate $\mathrm{D}$-galactose-induced aging in rats by activating FOXO3a and Nrf2 pathways. Oxid Med Cell Longev 2014: 320513, 2014.
19. Weng Y, Yu L, Cui J, Zhu YR, Guo C, Wei G, Duan JL, Yin Y, Guan Y, Wang YH, et al: Antihyperglycemic, hypolipidemic and antioxidant activities of total saponins extracted from Aralia taibaiensis in experimental type 2 diabetic rats. J Ethnopharmacol 152: 553-560, 2014

20. Li C, Liu Z, Tian J, Li G, Jiang W, Zhang G, Chen F, Lin P and Ye Z: Protective roles of Asperosaponin VI, a triterpene saponin isolated from Dipsacus asper Wall on acute myocardial infarction in rats. Eur J Pharmacol 627: 235-241, 2010.

21. Zhang JY, Chen ZW and Yao H: Protective effect of urantide against ischemia-reperfusion injury via protein kinase $\mathrm{C}$ and phosphtidylinositol 3'-kinase - Akt pathway. Can J Physiol Pharmacol 90: 637-645, 2012.

22. Koyama T, Temma K and Akera T: Reperfusion-induced contracture develops with a decreasing [Ca2+]i in single heart cells. Am J Physiol 261: H1115-H1122, 1991.

23. Haunstetter A and Izumo S: Apoptosis: Basic mechanisms and implications for cardiovascular disease. Circ Res 82: 1111-1129, 1998.

24. Yue TL, Wang C, Gu JL, Ma XL, Kumar S, Lee JC, Feuerstein GZ, Thomas H, Maleeff B and Ohlstein EH: Inhibition of extracellular signal-regulated kinase enhances Ischemia/ Reoxygenation-induced apoptosis in cultured cardiac myocytes and exaggerates reperfusion injury in isolated perfused heart. Circ Res 86: 692-699, 2000.

25. Hausenloy DJ and Yellon DM: Preconditioning and postconditioning: united at reperfusion. Pharmacol Ther 116: 173-191, 2007.

26. Maxwell SR and Lip GY: Reperfusion injury: a review of the pathophysiology, clinical manifestations and therapeutic options. Int J Cardiol 58: 95-117, 1997.

27. Zhang J, Lu S, Wang H and Zheng Q: Protective role of Aralia elata polysaccharide on mercury(II)-induced cardiovascular oxidative injury in rats. Int J Biol Macromol 59: 301-304, 2013.

28. Zhang J, Wang $\mathrm{H}$ and Zheng Q: Cardioprotective effect of Aralia elata polysaccharide on myocardial ischemic reperfusion (IR) injury in rats. Int J Biol Macromol 59: 328-332, 2013.

29. Zhang J, Wang H, Xue Y and Zheng Q: Cardioprotective and antioxidant activities of a polysaccharide from the root bark of Aralia elata (Miq.) Seem. Carbohydr Polym 93: 442-448, 2013.

30. Wang M, Xu X, Xu H, Wen F, Zhang X, Sun H, Yao F, Sun G and Sun X: Effect of the total saponins of Aralia elata (Miq) Seem on cardiac contractile function and intracellular calcium cycling regulation. J Ethnopharmacol 155: 240-247, 2014.

31. Mohanty IR, Maheshwari U, Joseph D and Deshmukh Y: Bacopa monniera protects rat heart against ischaemia-reperfusion injury: Role of key apoptotic regulatory proteins and enzymes. J Pharm Pharmacol 62: 1175-1184, 2010.

32. Ertracht O, Malka A, Atar S and Binah O: The mitochondria as a target for cardioprotection in acute myocardial ischemia. Pharmacol Ther 142: 33-40, 2014.

33. Crow MT, Mani K, Nam YJ and Kitsis RN: The mitochondrial death pathway and cardiac myocyte apoptosis. Circ Res 95: 957-970, 2004.

34. Xie Z, Koyama T, Suzuki J, Fujii Y, Togashi H, Sawa H and Nagashima K: Coronary reperfusion following ischemia: Different expression of bcl-2 and bax proteins, and cardiomyocyte apoptosis. Jpn Heart J 42: 759-770, 2001.

35. Armstrong JS: Mitochondria: A target for cancer therapy. Br J Pharmacol 147: 239-248, 2006.

36. Dorn GW II: Apoptotic and non-apoptotic programmed cardiomyocyte death in ventricular remodelling. Cardiovasc Res 81: 465-473, 2009.

37. Steinberg GR and Kemp BE: AMPK in Health and Disease. Physiol Rev 89: 1025-1078, 2009.

38. Zhang P, Hu X, Xu X, Fassett J, Zhu G, Viollet B, Xu W, Wiczer B, Bernlohr DA, Bache RJ, et al: AMP activated protein kinasealpha2 deficiency exacerbates pressure-overload-induced left ventricular hypertrophy and dysfunction in mice. Hypertension 52: 918-924, 2008.

39. Horman S, Beauloye C, Vanoverschelde JL and Bertrand L: AMP-activated protein kinase in the control of cardiac metabolism and remodeling. Curr Heart Fail Rep 9: 164-173, 2012.

40. Lopaschuk GD: AMP-activated protein kinase control of energy metabolism in the ischemic heart. Int J Obes 32 (Suppl 4): S29-S35, 2008.

41. Russell RR III, Li J, Coven DL, Pypaert M,Zechner C, Palmeri M, Giordano FJ, Mu J, Birnbaum MJ and Young LH: AMP-activated protein kinase mediates ischemic glucose uptake and prevents postischemic cardiac dysfunction, apoptosis, and injury. J Clin Invest 114: 495-503, 2004. 